\title{
Efeito da Irradiação Gama nas Propriedades Mecânicas e Térmicas de Redes DGEBA/Amina Cicloalifática com Potencial para Aplicações Médicas
}

\author{
Juliana C. Neves, Glaura G. Silva \\ Universidade Federal de Minas Gerais, UFMG \\ Marcio W. Duarte Mendes, Ana H. Bressiani, José C. Bressiani \\ Instituto de Pesquisas Energéticas e Nucleares, IPEN \\ Filiberto González Garcia \\ Universidade Federal de Itajubá, UNIFEI
}

\begin{abstract}
Resumo: Este trabalho apresenta uma caracterização de sistemas epoxídicos a base do éter diglicidílico do bisfenol A (DGEBA), curado com dois co-monômeros do tipo amina cicloalifática - piperidina (Pip) e 4,4'-diamino-3,3'dimetildiciclohexilmetano (3DCM) - antes e após a aplicação de irradiação com raios gama. Variações nas propriedades mecânicas e térmicas foram investigadas através de medidas do módulo elástico, da temperatura de transição vítrea e do perfil de degradação dos materiais. Foi possível observar que uma dose de irradiação de 50 kGy provoca variações sutis em propriedades como rigidez e estabilidade, que são relevantes do ponto de vista fundamental. Este trabalho permite afirmar que as variações de propriedades observadas não implicam em impacto negativo na utilização destes sistemas como biomaterial na área médica, considerando a etapa de esterilização por radiação de que são objeto.
\end{abstract}

Palavras-chave: Polímeros epoxídicos, raios gama, degradação, reticulação.

\section{Effect of Gamma Irradiation on Mechanical and Thermal Properties of DGEBA/ Cycloaliphatic Amine Networks with Potential for Medical Applications}

\begin{abstract}
Two epoxy polymers based on diglycidyl ether of bisphenol A (DGEBA), cured with piperidine (Pip) and 4,4'-diamino-3,3'-dimethyldicyclohexylmethane (3DCM), were characterized before and after treatment with $\gamma$ irradiation. The changes in the mechanical and thermal properties were studied by elastic modulus, glass transition temperature and degradation temperature measurements. A dose of $50 \mathrm{kGy}$ of irradiation caused subtle variations in properties such as rigidity and stability, which are relevant from the fundamental point of view. The variations do not imply on negative impact when considering the stage of sterilization during the use of these systems as a biomaterial in the medical area.
\end{abstract}

Keywords: Epoxy polymers, gamma rays, degradation, crosslinking.

\section{Introdução}

Existe na atualidade uma grande demanda por biomateriais que possam melhorar a qualidade de vida do ser humano ${ }^{[1-3]}$. Os polímeros epoxídicos apresentam propriedades otimizadas tais como dureza, tenacidade, resistência química, baixa contração, assim como excelente adesão, que são associadas à natureza estrutural dos monômeros que formaram a rede tridimensional ${ }^{[4,5]}$. Estes materiais poliméricos apresentam potencialidade para serem utilizados em algumas aplicações médicas, porque manifestam boas propriedades biológicas em paralelo às propriedades mecânicas adequadas ${ }^{[6-9]}$. Esta afirmação pode ser corroborada pelos estudos prévios do grupo com estes polímeros, usando ensaios biológicos in vitro de compatibilidade que mostraram reduzida atividade trombogênica com o sangue humano e não citotoxicidade com células de ovário hanster chinês ${ }^{[10]}$. Neste trabalho, os polímeros formulados com aminas cicloalifáticas mostraram melhor comportamento biológico, ou seja, reduzido comportamento trombogênico quando comparado às aminas alifáticas. Além disto, as aminas cicloalifáticas apresentam maior tempo de manipulação que as aminas alifáticas, o que é interessante para preparar formulações para o encapsulamento de nanopartículas ferromagnéticas, que são utilizadas no procedimento terapêutico de magnetohipertermia para o tratamento do câncer ${ }^{[11]}$. Outro uso onde o maior tempo de manipulação seria desejável é o revestimento de aço inoxidável utilizado em aplicações cardiovasculares como, por exemplo, stents vasculares e coronários, que estão sendo desenvolvidos pelo grupo nesta pesquisa.

A esterilização de biomateriais usando uma dose de irradiação de raios gama é uma técnica comumente utilizada $^{[12,13]}$. Esta técnica apresenta como vantagens alta

Autor para correspondência: Glaura Goulart Silva, Departamento de Química, Instituto de Ciências Exatas, Universidade Federal de Minas Gerais - UFMG, Av. Presidente Antônio Carlos, 6627, Pampulha, CEP 31270-901, Belo Horizonte, MG, Brasil, e-mail: glaura@qui.ufmg.br 
eficiência, efeitos térmicos negligenciáveis e não envolve o uso de substâncias tóxicas. Segundo a literatura, a dose de radiação padrão para a esterilização de dispositivos médicos corresponde a $25 \mathrm{kGy}^{[14]}$.

A resina sólida do tipo éter diglicidílico do bisfenol A (de massa molar média de $4 \times 10^{4}$ ), quando submetida a elevada dose de irradiação de $2 \mathrm{MGy}$, para aplicação como agente de solidificação em resíduos radioativos, pode sofrer degradação por quebra de ligações químicas e pode ocorrer também reticulação induzida pela irradiação ${ }^{[15]}$.

Existem possibilidades de utilizar a irradiação de raios gama para induzir reticulação em polímeros como, por exemplo, para: i) produzir copolímeros de polipropileno alfa-olefinas-metalocenos, usando dose de 10 a 100 kGy ${ }^{[16]}$; ii) melhorar as propriedades mecânicas de polietileno linear de baixa densidade (LLDPE), usando dose de 150 e 250 kGy ${ }^{[17]}$; iii) sintetizar copolímeros de enxerto de ácido acrílico na superfície de poliestireno extrudado $^{[18]}$; iv) e polimerizar resinas epoxídicas contendo iniciadores apropriados ${ }^{[19,20]}$.

A utilização de materiais poliméricos em campos de irradiação elevados requer um bom conhecimento de seu envelhecimento por irradiação induzida para um melhor aproveitamento a médio e longo prazos e para evitar riscos durante sua utilização. Vários estudos foram realizados sobre o efeito da irradiação usando feixe de elétrons ou raios gama em polímeros e, em particular, para polímeros epoxídicos usando compostos modelos ${ }^{[21-25]}$. A degradação em polímeros é um fenômeno visível pela presença de amarelamento, escurecimento e/ou fragilização e pode acarretar alterações nas propriedades mecânicas. A degradação é geralmente aumentada quando o material epoxídico está exposto a altas doses de irradiação gama (na ordem de 10 a 70 MGy), que leva a uma diminuição das propriedades mecânicas tais como; resistência à flexão $\left(\sigma_{\mathrm{R}}\right)$, tenacidade $\left(\mathrm{K}_{\mathrm{IC}}\right)$, temperatura de transição vítrea, etc. Segundo a literatura, a diminuição de $\mathrm{K}_{\mathrm{IC}}$ pode ocorrer por uma combinação de degradação e reticulação ${ }^{[24]}$.
Embora vários estudos tenham sido publicados sobre o efeito da irradiação para polímeros epoxídicos, no limite de nosso conhecimento, nenhum trabalho foi realizado sobre o efeito da irradiação de redes epoxídicas à base do éter diglicidílico do bisfenol A curado com aminas cicloalifáticas, usando baixas doses de irradiação de raios gama entre 25 e $50 \mathrm{kGy}$.

$\mathrm{O}$ objetivo deste trabalho foi, portanto, estudar o efeito da aplicação de irradiação de raios gama, usando uma fonte de ${ }^{60} \mathrm{Co}$ em dois polímeros epoxídicos do ponto de vista de mudanças nas propriedades mecânicas e térmicas e na análise morfológica da superfície dos corpos de prova. $\mathrm{O}$ presente estudo permitirá avaliar o impacto da metodologia de esterilização destes materiais em produtos para aplicações médicas. Para isto, foram selecionados dois polímeros, que foram formulados à base do monômero do tipo éter diglicidílico do bisfenol A (DGEBA) e dois co-monômeros do tipo amina cicloalifática a saber: piperidina (Pip) e a 4,4'-diamino-3,3' -dimetildiciclohexilmetano (3DCM), respectivamente. Estes dois sistemas apresentam valores de massas molares e funcionalidades dos co-monômeros bem diferentes (Tabela 1) e permitem estabelecer um quadro comparativo bastante geral, especialmente quando colocados em conjunto com o estudo prévio do grupo sobre os co-monômeros: dietilenotriamina (DETA), 1-(2-aminoetil)piperazina (AEP) e isoforona diamina (IPD), recentemente publicado ${ }^{[26]}$. Foram avaliadas as variações do módulo elástico, medido usando o método dinâmico de excitação por impulso, da temperatura de transição vítrea e da temperatura onde ocorre a taxa máxima de decomposição dos materiais. As discussões foram complementadas pela análise de microscopia eletrônica de varredura das superfícies dos materiais.

\section{Experimental}

\section{Materiais}

As formulações utilizadas estão baseadas no oligômero DGEBA, produto da reação de epicloridrina

Tabela 1. Estrutura química e algumas propriedades dos monômeros utilizados.

\begin{tabular}{|c|c|c|c|c|}
\hline Monômeros & Estrutura Química & Fornecedor & $\mathrm{M} / \mathrm{g} \mathrm{mol}^{-1}$ & $\mathbf{F}$ \\
\hline $\begin{array}{l}\text { Éter diglicidílico do } \\
\text { bisfenol A (DGEBA) }\end{array}$ & & $\begin{array}{c}\text { Dow Quimica } \\
\text { DER } 331\end{array}$ & $\sim 374$ & 2 \\
\hline Piperidina (Pip) & & Aldrich $(99,5 \%)$ & 85,15 & 1 \\
\hline \multirow[t]{2}{*}{$\begin{array}{c}\text { 4,4'-Diamino-3.3'- } \\
\text { dimetildiciclohexilmetano } \\
\text { (3DCM) }\end{array}$} & & Aldrich $(99,0 \%)$ & 238,41 & 4 \\
\hline & $\mathrm{CH}_{3}$ & & & \\
\hline
\end{tabular}

M: massa molar; F: funcionalidade. 
e bisfenol-A, produto técnico DER 331, produzido e comercializado pela Dow Química do Brasil S.A., com dois co-monômeros do tipo aminas, cicloalifáticas. A Tabela 1 apresenta a estrutura básica e algumas propriedades dos monômeros utilizados. A resina foi desidratada cuidadosamente por tratamento a vácuo a $80{ }^{\circ} \mathrm{C}$ até o desaparecimento das bolhas. Os co-monômeros foram utilizados como recebidos.

\section{Preparação dos polímeros epoxídicos}

O polímero à base de 3DCM foi preparado utilizando proporções estequiométricas (equivalentes de grupos epóxi/equivalentes de hidrogênios amina, e/a = 1). Isto foi baseado na determinação de grupos funcionais por métodos de titulação química ${ }^{[27,28]}$. A formulação preparada foi agitada à temperatura ambiente por 1 minuto para assegurar a dissolução do co-monômero e depois foi vazada em moldes de silicone com cavidades retangulares. O programa de cura usado foi em duas etapas. A primeira etapa, com 24 horas à temperatura ambiente, e a segunda, em condições isotérmicas, que permitem alcançar a conversão máxima, o que foi otimizado por estudos calorimétricos anteriores (DSC) ${ }^{[10,29]}$. Para o sistema com piperidina foi utilizada uma concentração de 5 phr (5 gramas por cada 100 gramas de resina). O programa de cura usado foi em duas etapas, a primeira com 30 minutos a $60{ }^{\circ} \mathrm{C}$, e a segunda com 16 horas a $120^{\circ} \mathrm{C}$. Os materiais após o programa de cura foram resfriados lentamente até chegar à temperatura ambiente. Então foram retirados do molde e usinados a seco até alcançar a forma de barras retangulares $(90,0 \mathrm{~mm}$ $\times 12,0 \mathrm{~mm} \times 3,3 \mathrm{~mm}$ ) para os ensaios mecânicos.

\section{Irradiação dos polímeros}

Os materiais foram irradiados à temperatura ambiente $\left(25^{\circ} \mathrm{C}\right)$ em atmosfera de ar com raios gama usando fonte de ${ }^{60} \mathrm{Co}$ em um equipamento Gammacell 220, e uma taxa de irradiação de $1,5 \mathrm{kGy} / \mathrm{h}$. As amostras foram irradiadas até alcançar a dose escolhida na série de $25 \mathrm{kGy}, 35 \mathrm{kGy}$, 40 kGy e 50 kGy, como descrito no trabalho prévio do grupo $^{[26]}$. Posteriormente as amostras foram retiradas da exposição.

\section{Medidas do módulo elástico}

Medidas de módulo elástico por meio do método dinâmico (MD) são baseadas nas frequências ressonantes dos materiais. O MD apresenta vantagens quando comparado com os métodos tradicionais por ser uma técnica não destrutiva e aplicada em amostras de geometria simples (paralelepípedos retangulares, cilindros e discos), além disso, o MD é um procedimento de fácil operação e baixo custo ${ }^{[30,31]}$.

As vibrações no método dinâmico são provocadas por excitação contínua ou impulso. Na técnica de excitação contínua as vibrações são induzidas continuamente nas amostras no modo de flexão. No segundo caso as vibrações são provocadas por meio de impactos leves. A frequência ressonante da amostra durante a vibração é determinada e utilizada para o cálculo do módulo elástico. Para barras retangulares o módulo elástico é dado pela Equação $1^{[32]}$ :

$$
E=0.9468\left(\frac{m f_{r}^{2}}{b}\right)\left(\frac{L^{3}}{t^{3}}\right) T
$$

onde $m$ é a massa do objeto, $f_{r}$ é a frequência ressonante, $b$ é a largura, $L$ o comprimento, $t$ é a espessura e $T$ é o fator de correção dado pela Equação 2:

$$
\begin{aligned}
T=1+6.858(1 & \left.+0.0752 \mu+0.8109 \mu^{2}\right)(t / L)^{2}-0.868(t / L)^{4} \\
& -\left[\frac{8.340\left(1+0.2023 \mu+2.173 \mu^{2}\right)(t / L)^{4}}{1+6.338\left(1+0.1408 \mu+1.536 \mu^{2}\right)(t / L)^{2}}\right]
\end{aligned}
$$

onde $\mu$ é o coeficiente de Poisson que foi considerado como $\mu=0,34$.

Um instrumento Grindosonic foi utilizado para realizar as medidas segundo o protocolo da norma ASTM $^{[32]}$. Para induzir vibração na barra retangular foi utilizada uma pequena esfera de polímero, presa a uma haste flexível, que foi aplicada diretamente no centro da amostra e a vibração foi detectada por meio de um microfone. As barras foram assentadas sobre tiras de poliuretano posicionadas nos pontos nodais, a $0,224 \mathrm{~L}$ de cada extremidade da barra retangular, onde L é o comprimento da amostra. $\mathrm{O}$ valor determinado do módulo foi o valor médio de 5 determinações.

\section{Calorimetria exploratória de varredura (DSC)}

A calorimetria exploratória de varredura (equipamento da TA Instruments, modelo DSC-Q1000) foi realizada com uma razão de aquecimento de $10{ }^{\circ} \mathrm{C} \mathrm{min}^{-1}$ em um intervalo de 20 a $200{ }^{\circ} \mathrm{C}$ em atmosfera de nitrogênio $\left(20 \mathrm{~cm}^{3} \mathrm{~min}^{-1}\right)$ com amostras de aproximadamente $12 \mathrm{mg}$. Para determinar a temperatura de transição vítrea $\left(T_{\mathrm{g}}\right)$ dos polímeros após a cura foram realizadas duas corridas de aquecimento a fim de verificar a possibilidade da existência de material residual não completamente reticulado. $\mathrm{O}$ valor de $T_{\mathrm{g}}$ foi determinado no início da mudança da capacidade calorífica em relação à linha base da curva de DSC (“on set").

\section{Análise termogravimétrica (TG)}

A decomposição térmica dos polímeros após o programa de cura e após serem submetidos a diferentes doses de irradiação foi avaliada usando analisador termogravimétrico (TA Instruments, modelo Q5000 IR), com uma taxa de aquecimento de $20^{\circ} \mathrm{C} \mathrm{min}-1$ a partir 30 até $700{ }^{\circ} \mathrm{C}$, em atmosfera de nitrogênio $\left(20 \mathrm{~cm}^{3} \mathrm{~min}^{-1}\right)$. Amostras de aproximadamente $10 \mathrm{mg}$ foram analisadas em cadinho de platina sem tampa. A temperatura onde ocorre a máxima taxa de decomposição do material é obtida no pico da curva derivada.

\section{Microscopia eletrônica de varredura (MEV)}

Imagens de MEV foram obtidas em equipamento Quanta 200, modelo FEG-FEI 2006, operando em vácuo sob feixe de elétrons com aceleração de $10 \mathrm{kV}$. As amostras foram recobertas com fina camada de ouro. 


\section{Resultados e Discussão}

\section{Propriedades mecânicas}

Na Tabela 2 estão sumarizadas as propriedades mecânicas dos polímeros sem irradiação e após serem submetidos a $50 \mathrm{kGy}$ de irradiação de raios gama. A tendência observada com o aumento da doses de irradiação é sistemática na série entre 25-50 kGy e apenas para a máxima dose as mudanças são mais significativas. Este comportamento também foi observado para outros sistemas em nosso trabalho anterior ${ }^{[26]}$. Portanto, neste trabalho privilegiou-se mostrar e discutir os resultados dos sistemas irradiados com a dose máxima de 50 kGy, a fim de evidenciar os efeitos limites observados.

O material epoxídico obtido com o endurecedor de maior funcionalidade 3DCM apresentou módulo elástico $25 \%$ maior que o material reticulado com Pip, o que é um resultado esperado. O comportamento mecânico observado para o material com Pip esta relacionado à estrutura particular desta rede que possui maior separação entre os pontos de entrecruzamento e maior tamanho das cadeias elasticamente ativas, quando comparada à rede com $3 \mathrm{DCM}^{[33,35]}$, o que significa menor densidade de reticulação e, como consequência disto, o material é mais flexível ${ }^{[33,36]}$.

Por outro lado, pode ser observado na Tabela 2 que o módulo elástico aumenta ligeiramente de $8 \%$ e $5 \%$ para os sistemas com Pip e com 3DCM respectivamente, quando a dose de irradiação é de $50 \mathrm{kGy}$. Este comportamento não era esperado, considerando que o aumento da dose de irradiação pode provocar o aumento da degradação do material, como resultado do aumento da quebra de ligações químicas. $\mathrm{O}$ aumento da quebra das ligações químicas pode induzir uma diminuição da densidade de reticulação ${ }^{[25]}$.

Segundo a literatura, a quebra de ligações químicas que ocorre nos polímeros epoxídicos quando submetidos a irradiação gama está relacionada às ligações entre os átomos de carbono-nitrogênio $(\mathrm{C}-\mathrm{N})$ e carbono-oxigênio (C-O), que apresentam um valor de entalpia média de ligação de $293 \mathrm{~kJ} / \mathrm{mol}$ e $358 \mathrm{~kJ} / \mathrm{mol}$, respectivamente. A quebra das ligações $\mathrm{C}-\mathrm{O}$ e $\mathrm{C}-\mathrm{N}$ originam grupos fenólicos e metilcetona terminais e, amina primária e secundária terminais, respectivamente ${ }^{[23]}$.

A irradiação em sua dose máxima induziu um aumento inesperado da rigidez para o qual deve-se procurar a explicação estrutural. Esta investigação será conduzida neste trabalho pela análise dos resultados de TG, DSC e imagens de microscopia.

Tabela 2. Módulo elástico obtido por meio das frequências flexionais. Dados para os sistemas epoxídicos antes de irradiar e após irradiação de 50 kGy de raios gama.

\begin{tabular}{ccc}
\hline \multirow{2}{*}{$\begin{array}{c}\text { Polímeros } \\
\text { epoxídicos }\end{array}$} & $\begin{array}{c}\text { Módulo elástico } \\
(\mathbf{G P a})\end{array}$ & $\begin{array}{c}\text { Módulo elástico } \\
(\mathbf{G P a})\end{array}$ \\
\cline { 2 - 3 } & $\mathbf{0 ~ k G y}$ & $\mathbf{5 0} \mathbf{~ k G y}$ \\
\hline DGEBA/PIP & $2,783 \pm 0,002$ & $3,012 \pm 0,001$ \\
DGEBA/3DCM & $3,462 \pm 0,010$ & $3,626 \pm 0,003$ \\
\hline
\end{tabular}

\section{Propriedades térmicas}

Nas curvas de derivada de TG (DTG), nas Figuras 1 e 2, observa-se que a temperatura onde ocorre a taxa máxima de degradação do polímero formulado com Pip é aproximadamente $75^{\circ} \mathrm{C}$ maior quando comparada ao sistema com 3DCM. Como será discutido a seguir, as temperaturas de transição vítrea dos dois sistemas são também muito diferentes, sendo que o sistema com Pip apresenta $\mathrm{Tg}$ inferior. O comportamento térmico aqui observado é consequência da formação de estruturas de rede bem diferentes, devido à diferença de funcionalidade entre Pip e 3DCM ${ }^{[34,35]}$, ver Tabela 1 . Os resultados das Figuras 1 e 2 mostram que a menor densidade de reticulação do sistema com Pip originou uma rede significativamente mais estável termicamente, quando comparada à rede formada com 3DCM.

A comparação do efeito da radiação sobre os dois polímeros epoxídicos permite afirmar que ocorrem variações sutis do perfil de degradação em função da temperatura (Figuras 1 e 2). A temperatura onde ocorre a taxa máxima de degradação desloca-se de aproximadamente $6{ }^{\circ} \mathrm{C}$ (Figura 1), quando há a aplicação da irradiação máxima para o sistema com Pip. No caso

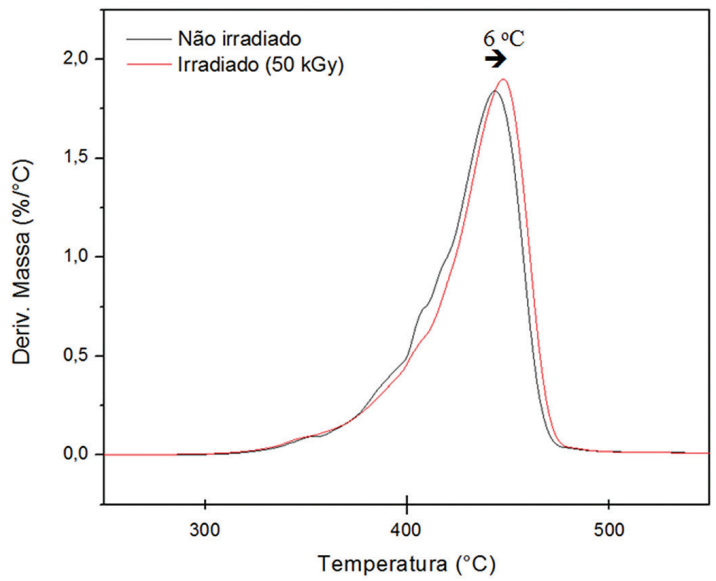

Figura 1. Curvas TG para epóxi/PIP não irradiado e após irradiação de 50 kGy.

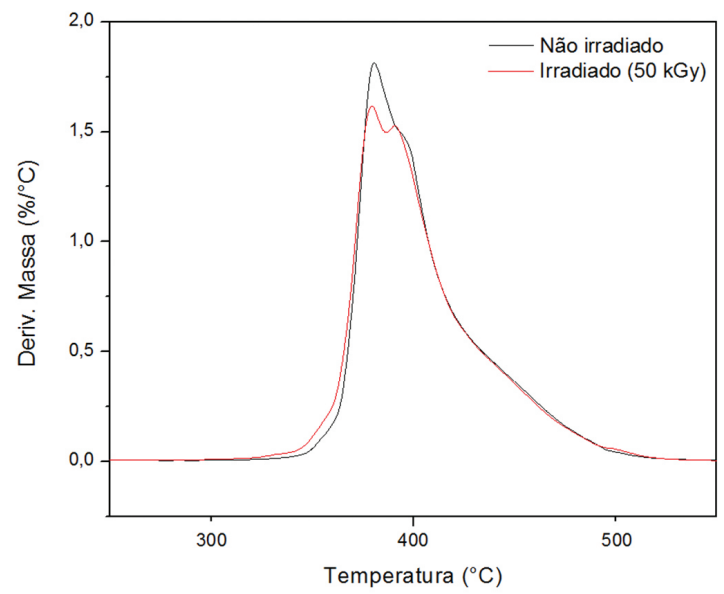

Figura 2. Curvas TG para epóxi/3DCM não irradiado e após irradiação de $50 \mathrm{kGy}$. 
do sistema com 3DCM, observa-se o desdobramento do pico da DTG (Figura 2) com uma tendência de um segundo pico localizar-se a temperaturas superiores. Estes resultados, juntamente com os dados de módulo elástico na Tabela 1 , indicam aumento sútil de rigidez e estabilidade térmica com a irradiação.

As Figuras 3 e 4 mostram as curvas DSC dos materiais analisados, que correspondem aos polímeros não irradiados e submetidos à irradiação de $50 \mathrm{kGy}$, formulados com Pip e 3DCM, respectivamente. Duas corridas de aquecimento foram realizadas, sendo que a primeira corrida informa sobre o comportamento térmico do material como preparado no laboratório e a segunda corrida fornece os dados de um sistema onde a história térmico-mecânica do material foi apagada.

A Tabela 3 apresenta os valores da temperatura de transição vítrea $\left(T_{\mathrm{g}}\right)$ dos polímeros obtidos por análises

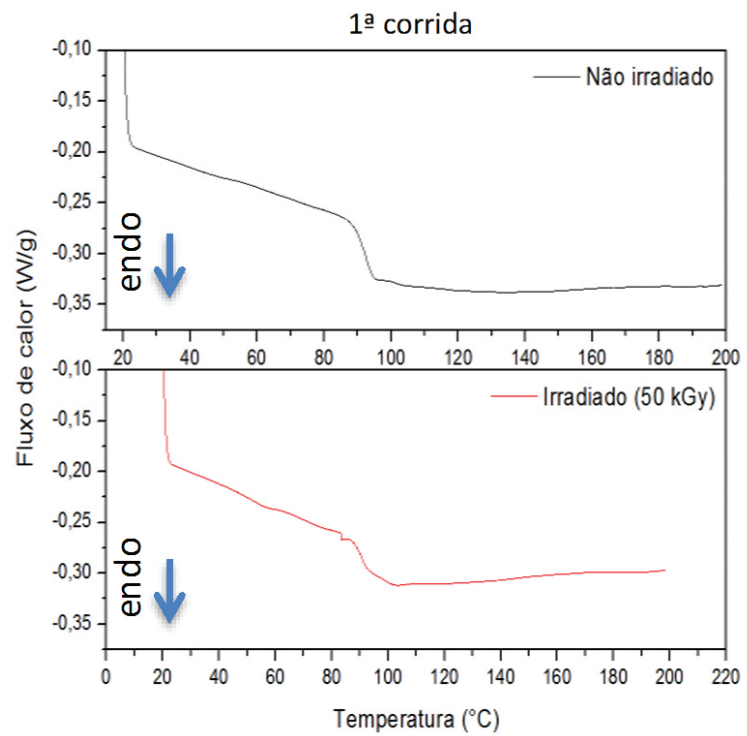

DSC. Pode ser observado que os polímeros com Pip e 3DCM apresentam uma diferença significativa entre seus valores de $\mathrm{Tg}$, devido às diferenças em densidade de reticulação, bem como características particulares de cada rede. Como já mencionado anteriormente, Pip

Tabela 3. Valores de temperatura de transição vítrea (Tg), para os polímeros epoxídicos antes e após a irradiação com 50 kGy, obtidos em duas corridas consecutivas de DSC.

\begin{tabular}{ccc}
\hline $\begin{array}{c}\text { Polímeros } \\
\text { epoxídicos }\end{array}$ & 1 $^{\text {a }}$ Corrida $\left({ }^{\circ} \mathbf{C}\right)$ & 2 ${ }^{\text {a }}$ Corrida $\left({ }^{\circ} \mathbf{C}\right)$ \\
\hline DGEBA/3DCM & 165 & 170 \\
DGEBA/Pip & 89 & 95 \\
\hline \multicolumn{3}{c}{ Após irradiação } \\
\hline DGEBA/3DCM & 158 & 161 \\
DGEBA/Pip & 88 & 94 \\
\hline
\end{tabular}

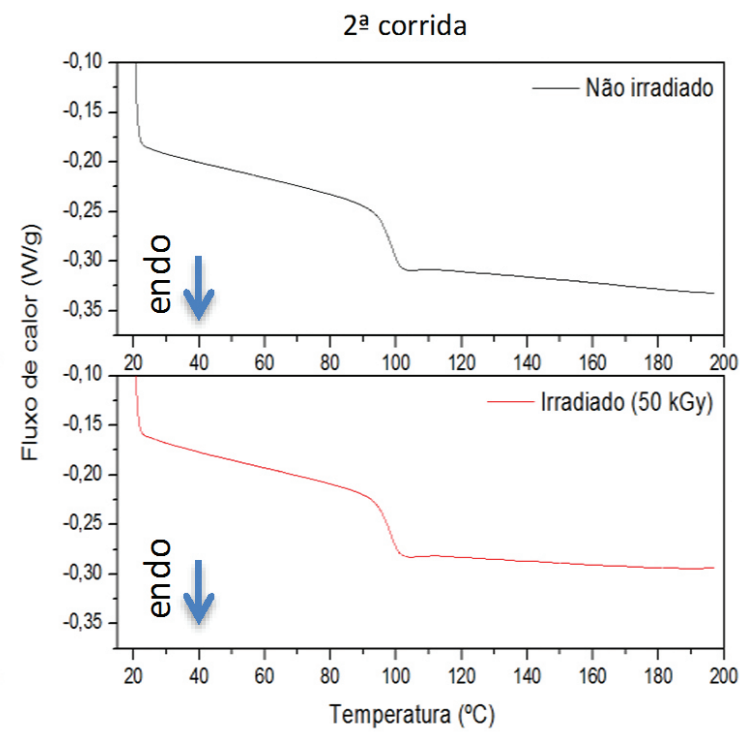

Figura 3. Curvas DSC para epóxi/PIP não irradiado e após irradiação de 50 kGy.
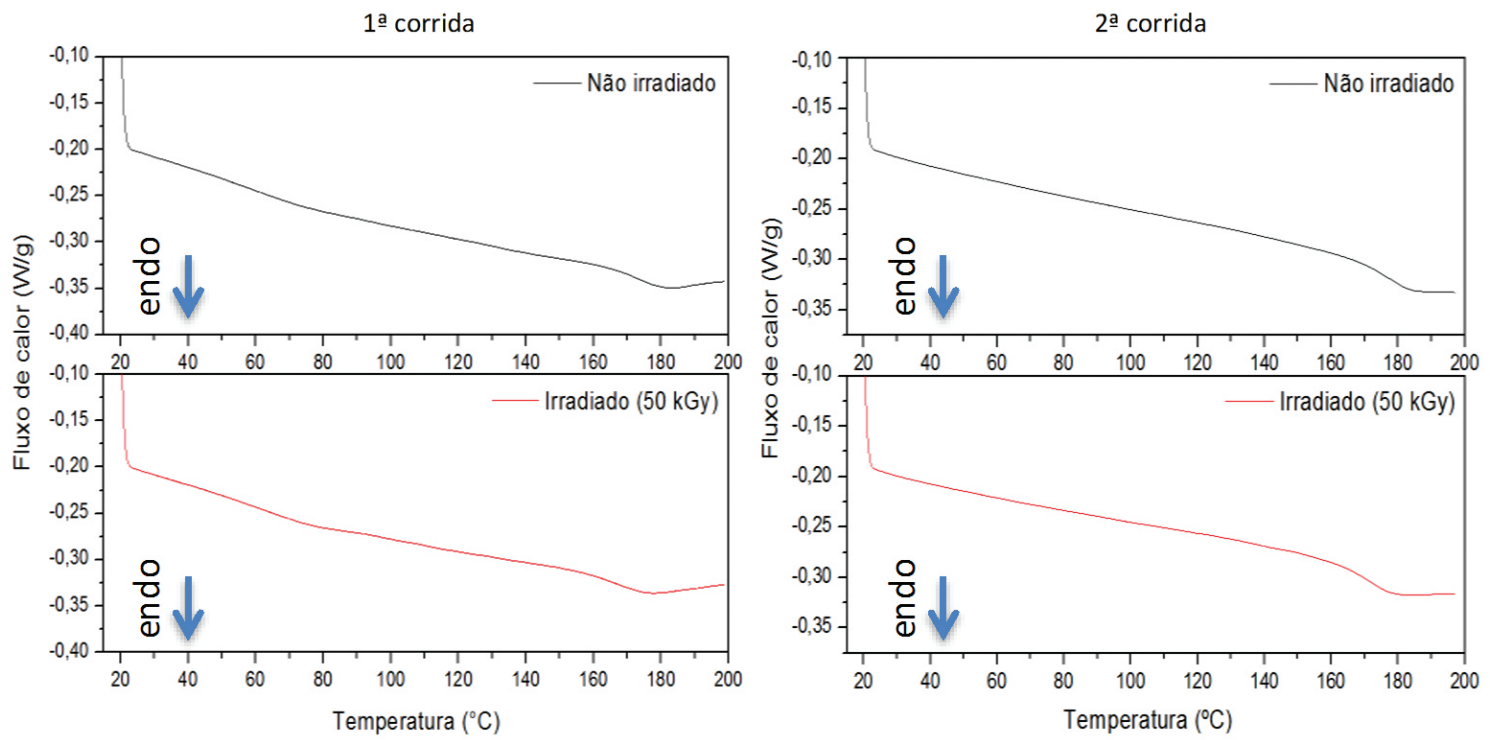

Figura 4. Curvas DSC para epóxi/3DCM não irradiado e após irradiação de 50 kGy. 
leva a materiais epoxídicos com menor densidade de reticulação $0^{[34,35]}$

O sistema com Pip mostra valores superiores em $6{ }^{\circ} \mathrm{C}$ para Tg na segunda varredura da DSC e estes são similares antes e após irradiação, considerando a precisão $\mathrm{de} \pm 1^{\circ} \mathrm{C}$. Este resultado parece indicar que o material com Pip é menos sensível ao efeito induzido pelos raios gama, embora os resultados de termogravimetria e módulo tenham evidenciado o oposto. O material baseado em 3DCM mostra um aumento de Tg na segunda corrida de $5{ }^{\circ} \mathrm{C}$ quando não irradiado (Tabela 3). Após a irradiação ocorre decréscimo de $\mathrm{Tg}$ para este sistema nas duas varreduras, o que indica que algum tipo de movimento segmentacional da cadeia polimérica foi acrescido, talvez por degradação induzida.

Os pequenos aumentos de Tg na segunda varredura de DSC (Tabela 3) informam que os dois sistemas apresentavam reatividade. Isto demonstra que as

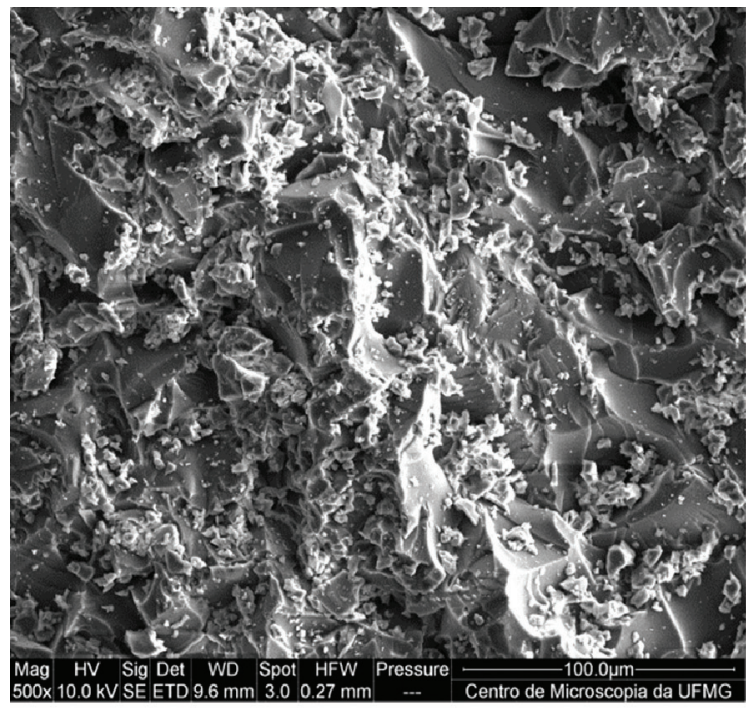

(a)

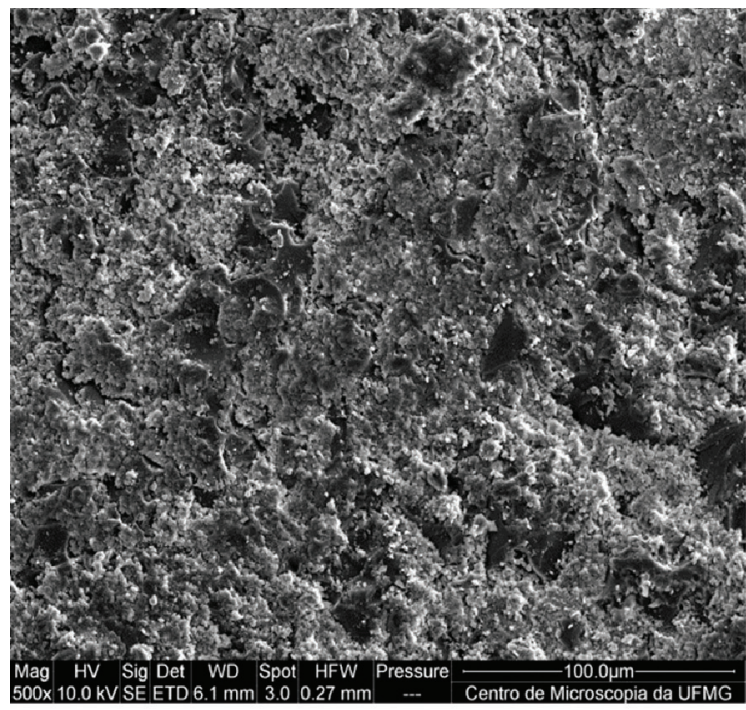

(c) condições de cura utilizadas não levaram a uma cura total para ambos os sistemas.

O estudo morfológico das superfícies dos corpos de prova de DGEBA/Pip e DGEBA/3DCM foi realizado com a produção de imagens em várias ampliações. Nas Figuras 5 e 6 são apresentadas imagens com duas ampliações para as superfícies dos corpos de prova antes e após irradiação com 50 kGy, que são representativas da morfologia dos materiais. Pode-se afirmar que o efeito da irradiação favorece o aumento no número de grânulos dispersos na superfície, ao mesmo tempo em que ocorre uma diminuição da diferença de altura entre os picos e vales da topografia. É como se a irradiação tivesse varrido a superfície aplainando-a por quebra dos picos mais altos. Este efeito é mais notável para o sistema com Pip (Figura 5).

O conjunto de propriedades estudado neste trabalho foi resumido na Figura 7. O aumento de rigidez e de

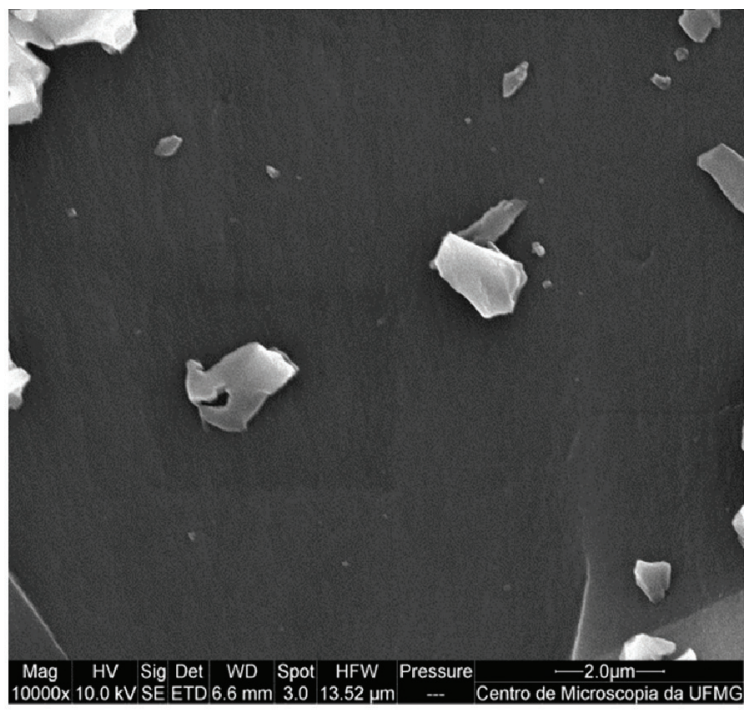

(b)

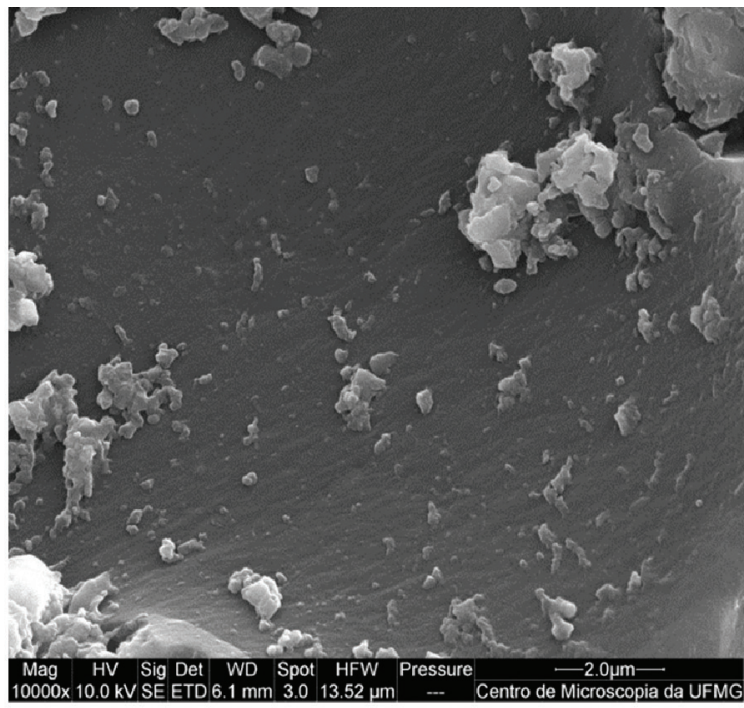

(d)

Figura 5. Micrografias (MEV) de superfícies para epóxi/PIP não irradiados (a, b) e após irradiação de 50 kGy (c, d). 


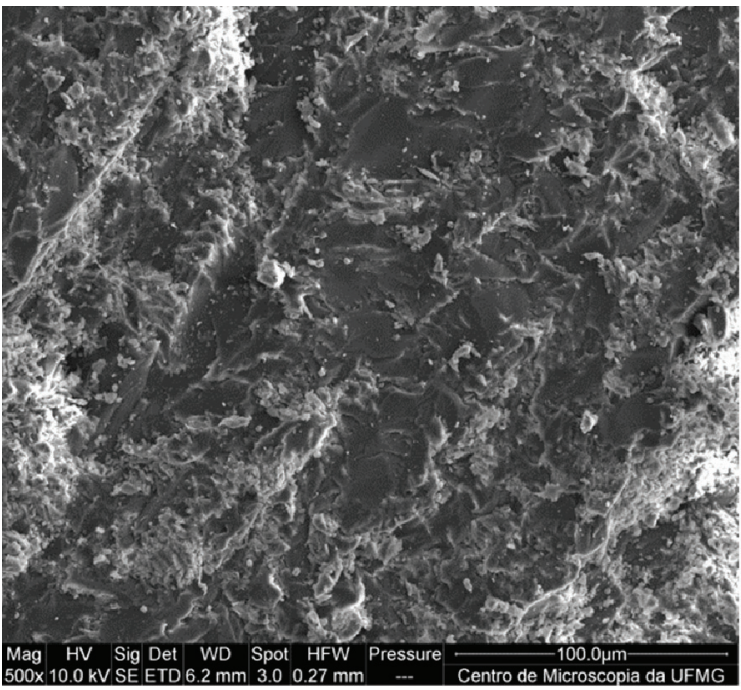

(a)

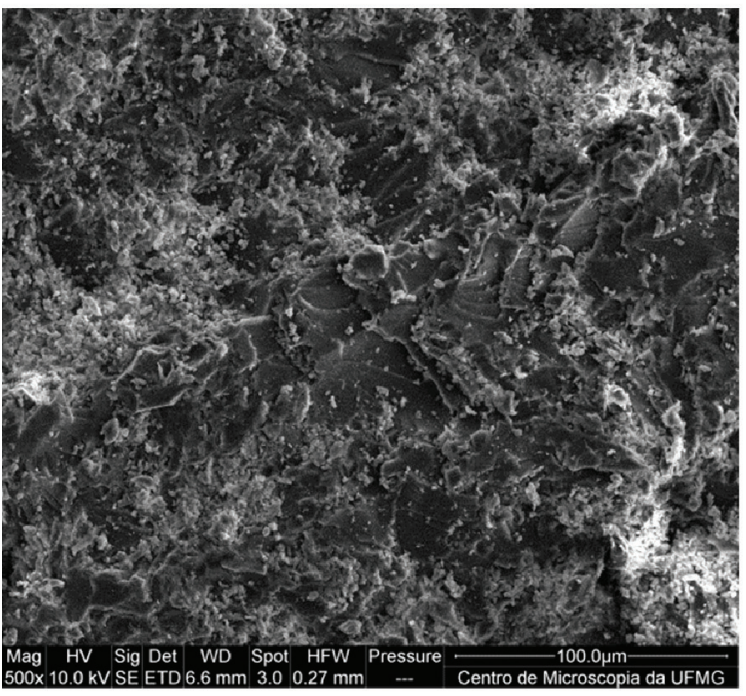

(c)

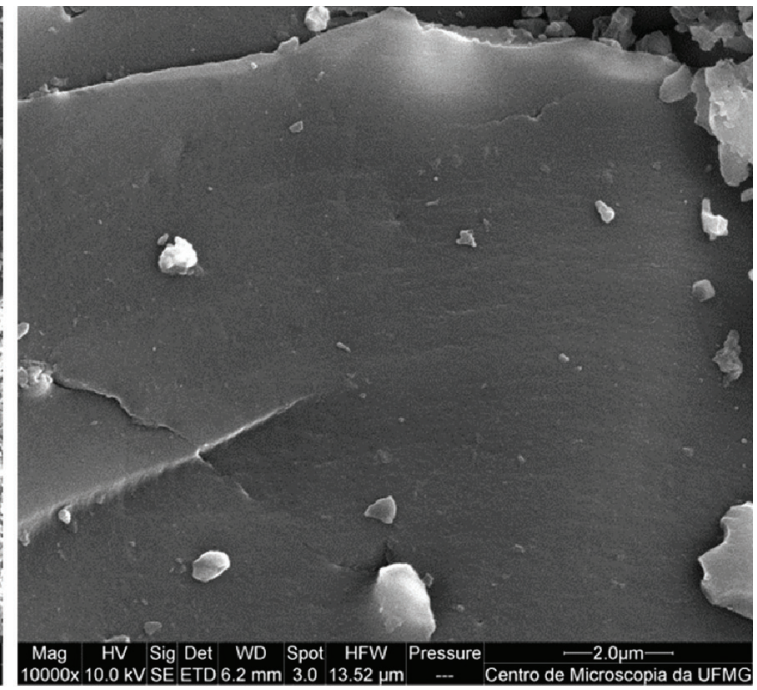

(b)

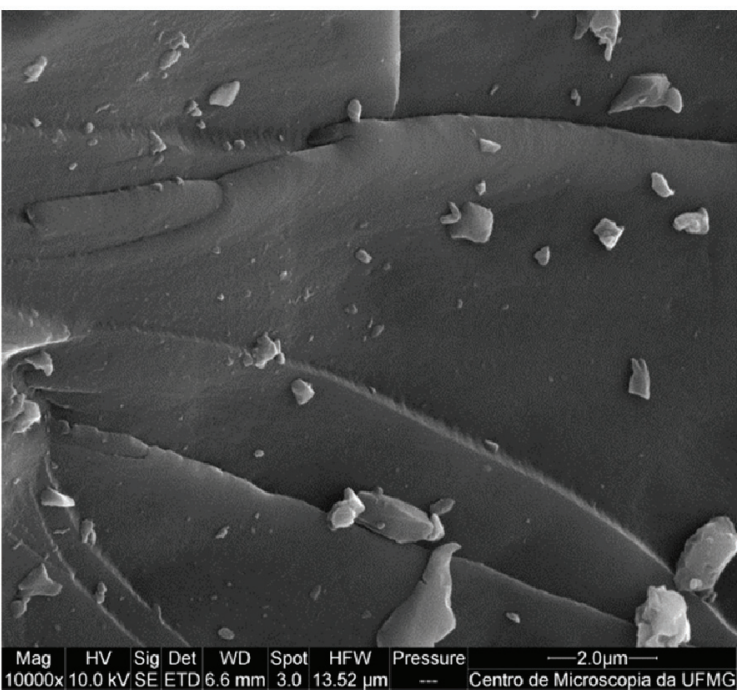

(d)

Figura 6. Micrografias (MEV) de superfícies para epóxi/3DCM não irradiados (a, b) e após irradiação de 50 kGy (c, d).

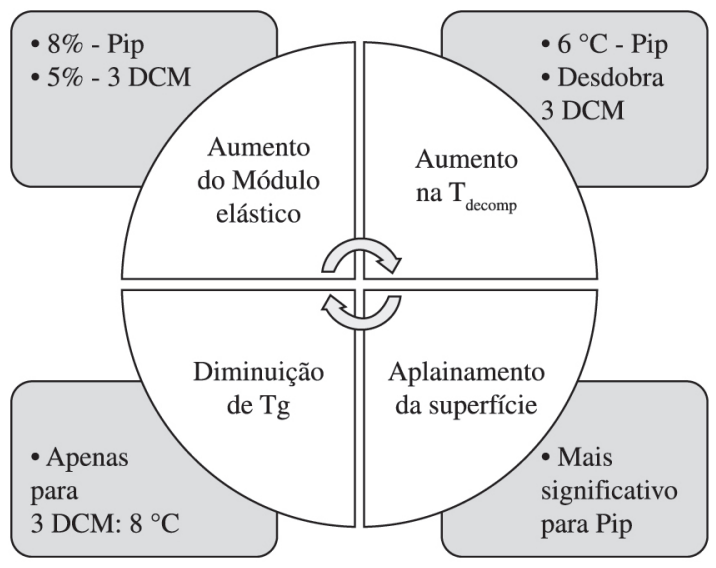

Figura 7. Resumo das variações observadas nas propriedades dos sistemas epoxídicos após irradiação com 50 kGy de radiação gama. (Onde está indicado Pip ou 3DCM entenda-se: sistema epoxídico com Pip ou 3DCM). temperatura de decomposição pode ser consequência de aumento de densidade de reticulação dos materiais após irradiação. Entretanto, para o sistema com 3DCM houve um decréscimo de Tg não desprezível após a irradiação, o que pode indicar degradação. Além disso, no caso da Pip ocorreu uma maior alteração da morfologia da superfície. Esta observação pode estar associada à quebra de ligações químicas ou degradação. Portanto, tem-se um quadro complexo na interpretação dos dados na Figura 7, que aponta para uma competição entre aumento de reticulação em paralelo à ocorrência de degradação sob efeito de irradiação. Este comportamento coincide com o divulgado na literatura, que menciona para materiais epoxídicos submetidos à irradiação de raios gama, a ocorrência simultânea de reticulação e degradação ${ }^{[15,24]}$.

Deve-se ressaltar que, a discussão deste trabalho até aqui focou no efeito da aplicação de irradiação de $50 \mathrm{kGy}$, quando foi possível observar variações não negligenciáveis nos parâmetros medidos. No caso de 
irradiação dentro do limite usualmente aplicado para o processo de esterilização no campo médico (25 kGy), as medidas experimentais mostram alterações abaixo do limite de precisão das técnicas de medida, o que pode ser um indicativo de que a esterilização é segura e não altera as características dos materiais. Entretanto, o processo de esterilização repetitivo e com pouco controle da intensidade da radiação gama pode gerar as alterações aqui observadas.

\section{Conclusões}

A irradiação com raios gama dos polímeros formulados com o éter diglicidílico do bisfenol A (DGEBA) e com piperidina (Pip) ou 4,4'-diamino3,3'-dimetildiciclohexilmetano (3DCM) como sistemas independentes, levou a mudanças no módulo elástico e nas propriedades térmicas. A dosagem máxima de 50 kGy provocou pequenos aumentos de rigidez e de estabilidade térmica, além de alterações na morfologia da superfície e de transição vítrea. Estas variações foram discutidas no contexto de reações competitivas de degradação e de reticulação.

Por outro lado, os valores de propriedades dos sistemas epoxídicos submetidos a doses de irradiação de raios gama da ordem de $25 \mathrm{kGy}$ não indicaram alterações significativas dentro da precisão das técnicas. Estes resultados são relevantes do ponto de vista aplicado, considerando a esterilização dos materiais epoxídicos usando a dose mínima de $25 \mathrm{kGy}$.

\section{Agradecimentos}

Os autores agradecem o apoio financeiro das entidades brasileiras CAPES, CNPq (Processo 304586/2011-0) e bolsa e FAPEMIG (Processo APQ 01736/11).

\section{Referências Bibliográficas}

1. Fernandes, I. L. B.; De Souza, D. C.; Coelho, S. L.; Chen, L. C. \& Guillo, L. A.- Toxicol. in Vitro, 27, p.198 (2013).

2. Panta, H. R.; Prabodh R.; Parka, C. H.; Tijing, L. D.; Jeong, Y. J. \& Kima, C. S. - Colloids Surf., B Biointerfaces, 102, p.152 (2013).

3. Sionkowska, A. - Prog. Polym. Sci., 36, p.1254 (2011). http://dx.doi.org/10.1016/j.progpolymsci.2011.05.003

4. Augustsson, C. - "NM Epoxy Hadbook", 3rd ed., Nils Malmgreen AB, (2004).

5. May, C. A. - "Epoxy Resins. Chemistry and Technology", 2nd ed, Marcel Dekker (1988).

6. Hsu, S. H.; Rung-Shu Chen, R. S.; Chang, Y. L.; Min-Huey Chen, M. H.; Kuo-Chung Cheng, K. C. \& Su, W. F. - Acta Biomater., 8, p.4151 (2012). PMid:22842038. http://dx.doi. org/10.1016/j.actbio.2012.07.030

7. Kim, T. G.; Lee, Y. H.; Bhattari, G.; Lee, N. H.; KwangWon Lee, K. W.; Ho-Keun Yi, H. \& K.; Yu, M. K. - Arch. Oral Biol. (2012). In press.

8. Huang, F. M. Ming-Yung Choub, M. Y. \& Chang, Y. C. - Biomaterials, 24, p.1869 (2003). http://dx.doi. org/10.1016/S0142-9612(02)00584-7

9. Van Wachem, P. B.; Brouwer, L. A.; Zeeman, R.; Dijkstra, P. J.; Feijen, J.; Hendriks, M.; Cahalan, P. T.;
Marja J. A. \& Van Luyn, M. J. - J. Biomed. Mater. Res., 53, p.19 (2000). http://dx.doi.org/10.1002/(SICI)10974636(2000)53:1<18::AID-JBM3>3.0.CO;2-J

10. González Garcia, F.; Leyva, M. E.; De Queiroz, A. A. A. \& Higa, O. Z. - J. Appl. Polym. Sci., 112, p.1215 (2009).

11. Castro, V. C.; Celestino, J.; De Queiroz A. A. A.; \& González Garcia, F. - Rev. Bras. Fis. Med., 4, p.79 (2010).

12. Soliman, Y. S.; Beshir, B. B.; Abdel-Fattah, A. A. \& Abdel-Rehim, F. - Appl. Radiat. Isotopes, 71, p.21 (2013). PMid:23079487. http://dx.doi.org/10.1016/j. apradiso.2012.09.016

13. Singh, D. \& Singh, R. - Radiat. Phys. Chem., 81, p.1781 (2012). http://dx.doi.org/10.1016/j. radphyschem.2012.06.010

14. Kowalski, J. B. \& Morrissey, R. F. - "Sterilization of implants", in: Biomaterials Science. An Introduction to Materials in Medicine, p.415, Ratner B.D., Hoffman A.S., Schoen, F.J.; Lemons, J.E. (eds), Academic Press, San Diego 1996.

15. Özdemir, T. \& Usanmaz, A. - Radiat. Phys. Chem., 77, p.299 (2008).

16. Satti, A. J.; Andreucetti, N. A.; Quijada, R. \& Valle, E. M. - Radiat. Phys. Chem., 81, p.1874 (2012). http://dx.doi. org/10.1016/j.radphyschem.2012.07.007

17. Krupa, I. \& Luyt, A. S. - Polym. Degrad. Stabil., 71, p.361 (2001). http://dx.doi.org/10.1016/S0141-3910(00)00186-5

18. Viorel Fugaru, V.; Bubueanu, G. \& Tuta, C. - Radiat. Phys. Chem. (2012). In press.

19. Alessi, S.; Parlato, A.; Dispenza, C.; De Maria, M. \& Spadaro, G. - Radiat. Phys. Chem., 76, p.1347 (2007). http://dx.doi.org/10.1016/j.radphyschem.2007.02.029

20. Nho, Y. C.; Kang, P. H. \& Park, J. S. - Radiat. Phys. Chem., 71, p.241 (2004).

21. Vignoud, L.; David, L.; Sixou, B.; Vigier, G \& Stevenson, I. - Nuclear Inst. Meth. Phys. Res. B., 185, p.336 (2001). http://dx.doi.org/10.1016/S0168-583X(01)00758-3

22. Vignoud, L.; David, L.; Sixou, B. \& Vigier, G. - Polymer, 42, p.4657 (2001). http://dx.doi.org/10.1016/S00323861(00)00791-6

23. Benard, F.; Campistron, I.; Laguerre, A. \& Laval, F. - Polym. Degrad. Stabil., 91, p.2110 (2006). http:// dx.doi.org/10.1016/j.polymdegradstab.2006.01.022

24. Devanne, T.; Brya, A.; Audouinb, L. \& Verdub, J. - Polymer, 46, p.229 (2005). http://dx.doi.org/10.1016/j. polymer.2004.07.039

25. Benard, F.; Buzaré, J. Y.; Campistron, I.; Lagurre, A. \& Laval, F. - Polym. Degrad. Stabil., 92, p.785 (2007). http:// dx.doi.org/10.1016/j.polymdegradstab.2007.02.005

26. Mendes, M. W. D.; Neves, J. C.; Bressiani A. H.; Bressiani, J. C. \& González Garcia, F. - Rev. Iber. Polim., 14, p.1 (2013).

27. González Garcia, F.; Da Silva, P. M.; Soares, B. G. \& Rieumont, J. - Polym. Test, 26, p.95 (2007). http://dx.doi. org/10.1016/j.polymertesting.2006.09.001

28. American Society for Testing and Materials - "ASTM D 1652: Standard test methods for epoxy content of epoxy resins", ASTM (1997).

29. González Garcia, F.; Miguez, E. \& Soares B. G. - Polímeros, 15, p.261 (2005). http://dx.doi.org/10.1590/S010414282005000400010 
30. Atri, R. R.; Ravichandran, K. S. \& Jha, S. K. - Mat. Sci. Eng. A, 271, p.150 (1999). http://dx.doi.org/10.1016/ S0921-5093(99)00198-7

31. Tognana, S.; Salgueiro, W.; Somoza, A. \& Marzocca, A. - Mat. Sci. Eng. A, 527, p.4619 (2010). http://dx.doi. org/10.1016/j.msea.2010.04.083

32. American Society for Testing and Materials - "ASTM E1876: Standard test method for dynamic Young's modulus, shear modulus and Poisson's ratio by impulse excitation of vibrations", ASTM (1997).

33. González Garcia, F.; Soares, B. G.; Pita, V. J. R. R.; Sánchez, R. \& Rieumont, J. - J. Appl. Polym. Sci., 106, p.2047 (2007).
34. Cuadrado, T. R.; Almaraz, A. \& Williams, R. J. J. - "Curing of Epoxy Resins with Piperidine”, in: Crosslinked Epoxies, p.170, B. Sedláček, J. Kahovec (eds.), De Gruyter, Berlin (1987).

35. Galego, N.; Vázquez, A. \& Williams, R. J. J. - Polymer, 35, p.857 (1994). http://dx.doi.org/10.1016/00323861(94)90886-9

36. González Garcia, F.; Leyva, M. E. \& De Queiroz, A. A. A. - Polímeros, 21, p.253 (2011). http://dx.doi.org/10.1590/ S0104-14282011005000057

Enviado: $22 / 01 / 13$

Reenviado: $29 / 03 / 13$

Aceito: 01/04/13 